\title{
Utilização do serviço de urgência pediátrica: a experiência de um centro português
}

\author{
Utilization of the pediatric emergency department: the experience of a Portuguese center
}

\author{
Margarida S. Rafael ${ }^{1} \bowtie$, Sofia Lopes Portela ${ }^{2}$, Paulo Sousa ${ }^{3}$, Adalberto Campos Fernandes ${ }^{3}$ \\ 1 Centro Hospitalar Barreiro-Montijo. Barreiro, Portugal. \\ 2 ISCTE Business School, Departamento de Métodos Quantitativos para Gestão e Economia. Lisboa, Portugal. \\ ${ }^{3}$ Universidade Nova de Lisboa, Escola Nacional de Saúde Pública. Lisboa, Portugal.
}

\section{RESUMO}

Objetivos: A elevada afluência aos serviços de urgência pediátrica tem gerado muita preocupação entre profissionais de saúde e administradores hospitalares. O objetivo deste estudo foi compreender as características da afluência a um serviço de urgência pediátrica e verificar se existe utilização injustificada do mesmo.

Métodos: Realizou-se um estudo retrospectivo no qual foram analisados todos os episódios de urgência pediátrica ocorridos durante o ano de 2012 em um hospital distrital da área metropolitana de Lisboa, Portugal. Os dados foram obtidos pelo serviço de informática do hospital, já estando os episódios classificados segundo o sistema de triagem de Manchester adaptado para Portugal - emergente, muito urgente, urgente, pouco urgente, não urgente e não classificável, o qual é atribuído a cada doente no momento de entrada no serviço de urgência pediátrica. Definiuse como episódio de urgência não justificado todos aqueles classificados como pouco urgentes e não urgentes. Os dados foram trabalhados de forma totalmente anônima, por meio do programa IBM SPSS Statistics e utilizando os testes estatísticos qui-quadrado e ANOVA one-way, com nível de significância de $5 \%(\mathrm{p}<0,05)$.

Resultados: Foi analisada uma amostra de 37.099 episódios de atendimento no serviço de urgência pediátrica, dos quais 19.478 (53\%) corresponderam a indivíduos do sexo masculino. A mediana de idade foi 4 anos (intervalo interquartil 1-9) e 78,4\% tinham até 10 anos. De todos os episódios, $98,8 \%$ foram classificados entre três categorias: muito urgentes, urgentes e pouco urgentes, sendo $15.470(41,7 \%)$ urgentes ou muito urgentes e 21.177 (57,1\%) pouco urgentes. Destes pacientes, 27.294 (73,6\%) recorreram ao serviço de urgência durante a semana e $28.679(77,3 \%)$ entre as 10 e as 24 horas. Verificou-se que em 90,8\% dos episódios muito urgentes, 97,1\% dos urgentes e 99,4\% dos pouco urgentes os pacientes tiveram alta sem necessitar de hospitalização.

Conclusões: Mais de metade das crianças que utilizaram o serviço de urgência pediátrica do hospital em estudo apresentavam situações pouco urgentes e praticamente todas essas tiveram alta para o domić́lio, com indicação para seguimento pelo médico assistente. Grande parte dos atendimentos ocorreu durante o horário de funcionamento dos centros de saúde.

DESCRITORES: medicina de urgência; serviços médicos de emergência; pediatria; uso excessivo de produtos e serviços de saúde.

\section{ABSTRACT}

Aims: The excess demand for pediatric emergency services has caused much concern among health professionals and hospital administrators. The aim of this study was to assess the utilization of a pediatric emergency department and to determine whether its use was injudicious.

Methods: Retrospective cross-sectional analysis of all emergency cases treated throughout 2012 in a general hospital located in the metropolitan area of Lisbon, Portugal. The data were obtained from the hospital's computer information system. Each patient was submitted at admission to the Manchester triage system adapted for Portugal, and the episodes were categorized into immediate, very urgent, urgent, standard, nonurgent, and not classified. All those episodes classified as standard and non-urgent were denoted as unjustified urgent episodes. The data were collected anonymously and analyzed by the IBM SPSS Statistics software using the chi-square test and one-way ANOVA at a $\%$ significance level $(\mathrm{p}<0.05)$.

Results: We analyzed 37,099 pediatric emergency department episodes, of which 19,478 patients were male (53\%), the median age was 4 years (interquartile range of $1-9$ years), and $78.4 \%$ were up to 10 years old. Of all the episodes, $21,177(57.1 \%)$ were classified as standard and $15,470(41.6 \%)$ as urgent or very urgent. Of these patients, 27,294 (73.6\%) used the emergency department during the week and 28,679 (77.3\%) between 10 a.m. and 12 a.m. It was found that in $90.8 \%$ of very urgent, $97.1 \%$ of urgent, and $99.4 \%$ of standard episodes, patients were discharged without the need for hospitalization.

Conclusions: More than half of the children who used the pediatric emergency department had standard or non-urgent needs, and almost all of them were discharged with follow-up recommendations by the attending physician. Most of the episodes occurred during opening hours of primary healthcare centers.

KEY WORDS: emergency medicine; emergency health services; pediatrics; overutilization of health services. 
Abreviaturas: SUP, serviços de urgência pediátrica; MCDT, meios complementares de diagnóstico e terapêutica.

\section{INTRODUÇÃO}

O fenômeno dos serviços de urgência pediátrica (SUP) colapsados não é recente e muita preocupação tem gerado ao longo dos tempos, não só a profissionais de saúde mas também a administradores e governantes. Porém, a sua resolução não é linear, por existirem poucos estudos sobre o que realmente se passa e quais as medidas mais adequadas a adotar [1]. Em Portugal, este fenômeno foi posto ainda mais em evidência desde 2010, quando o atendimento da idade pediátrica foi alargado até aos 18 anos [2,3].

A grande afluência aos SUP pode comprometer o atendimento a vários níveis, uma vez que os torna morosos, podendo provocar atrasos no atendimento a verdadeiras urgências, levando também à utilização indevida de recursos humanos e materiais, em situações que deles não necessitariam. Isso dá origem a uma cascata de descontentamento e ansiedade, tanto nos pacientes como nos profissionais de saúde, assim como inevitavelmente sobrecarrega os orçamentos hospitalares [4]. Em alguns estudos [1,5] foi possível estabelecer relação entre serviços de urgência superlotados, sobretudo às custas de situações não urgentes, e tempos de espera prolongados, o que provoca efeitos nefastos na qualidade do atendimento, na utilização dos recursos e nos custos a eles associados.

Portanto, é importante apurar a realidade hospitalar para poder criar soluções adequadas às necessidades dos doentes, mas que ao mesmo tempo respeitem a cadeia de atendimento, utilizando os recursos de acordo com as necessidades: cuidados de saúde primários para doenças agudas não urgentes e cuidados diferenciados para quadros clínicos que efetivamente deles necessitem [6,7].

Por outro lado, é importante conhecer a realidade epidemiológica do que se passa nos serviços de prestação de cuidados de saúde, tanto numa perspetiva presente como futura, para melhor adequar os recursos às necessidades $[8,9]$. Assim, poderão ser tomadas medidas que busquem alterar os hábitos de conduta, tendo sempre em conta a realidade da população à qual se aplicam as mesmas, e que visem sobretudo a aplicabilidade certa para que se consiga manter um atendimento de excelência com os recursos disponíveis $[9,10]$.

Nesse contexto surgiu este estudo, com o objetivo principal de saber se existe ou não utilização injustificada do SUP, mas também com o intuito de compreender as características da afluência ao mesmo. Apesar de incidir sobre uma população restrita e específica, visou o conhecimento de uma realidade, com o objetivo de contribuir para uma visão global do que se passa em hospitais com as mesmas características, pretendendo assim encontrar medidas que proporcionem melhor qualidade e custo-benefício na gestão dos recursos dos SUP.

\section{MÉTODOS}

Foi realizado um estudo observacional retrospectivo num SUP de um hospital geral e distrital da Área Metropolitana de Lisboa, Portugal. Em Portugal, todos os hospital integram uma rede hierarquizada de estabelecimentos hospitalares, definindo-se como um hospital geral e distrital aquele que possui serviço de urgência, ambulatórios e serviços de meios complementares de diagnóstico e terapêutica (MCDT), completando ainda a função assistencial com o ensino e eventual participação em investigação científica [11-13].

A amostra em estudo foi constituída por todos os episódios de urgência pediátrica ocorridos nesse hospital durante um ano, entre 01 de janeiro e 31 de dezembro de 2012. Todos os atendimentos de urgência pediátrica foram incluídos, não existindo critérios de exclusão. Em Portugal a idade pediátrica é definida entre os 0 dias e os 17 anos e 364 dias de vida [2].

Os dados foram obtidos pelo serviço de informática do hospital, com base no programa de urgência pediátrica SIRIU $^{\circledR}$. Os episódios atendidos no SUP foram registados de forma totalmente anônima sem consulta aos processos clínicos, de forma que durante o período de estudo alguns indivíduos podem ter gerado mais que um episódio.

O estudo obteve aprovação da Comissão Nacional de Protecção de Dados Portuguesa e da Direção do Centro Hospitalar Barreiro Montijo, EPE, em 2012. Constituiu ainda fonte de informação para este estudo, toda aquela que é oficial, pública e se encontra disponível online através das páginas eletrônicas do Ministério da Saúde, Direcção Geral de Saúde, Instituto Nacional de Estatística e Administração Regional de Saúde de Lisboa e Vale do Tejo.

Os episódios foram agrupados em categorias, de acordo com o sistema de triagem de Manchester, as quais se caracterizam por cor, número, tipo de urgência e tempo máximo de espera para uma primeira avaliação médica em minutos. O vermelho corresponde aos 
episódios de emergência; laranja aos muito urgentes; amarelo aos urgentes; verde aos pouco urgentes; azul aos não urgentes [14]. A categoria constava dos registos obtidos, sendo que quando os dados foram recolhidos pelo serviço de informática, os atendimentos já vinham todos classificados. A classificação é atribuída por um enfermeiro após a chegada da criança ao SUP, em pequena entrevista, seguindo o algoritmo de atribuição de classificação oficial em vigor para o SUP em estudo. Definiu-se como episódio de urgência não justificado todos aqueles classificados como pouco urgentes ou não urgentes segundo o sistema de triagem de Manchester adaptado. Em Portugal, esse sistema incorpora seis categorias, mais uma do que o original. A categoria adicionada é a não classificável, à qual se atribui a cor branca e que corresponde a situações de atividade programada ou não urgente [15] (Figura 1).

Foram investigadas as relações entre a categoria do episódio e um conjunto de variáveis como idade, sexo, dia da semana e hora do episódio de urgência, realização de MCDT e tipo de saída. Para o estudo das relações entre a categoria do episódio e as variáveis sexo, dia da semana, hora do episódio, MCDT e tipo de saída, realizaram-se o teste qui quadrado e respectivo ajuste residual, considerando-se um nível de significância de $5 \%(\mathrm{p}<0,05)$. Para o estudo da relação entre a categoria do episódio e a variável idade utilizou-se o teste ANOVA oneway e um teste de comparação múltipla com um nível de significância de $5 \%(\mathrm{p}<0,05)$. O tratamento estatístico dos dados foi realizado por meio do programa IBM SPSS Statistics versão 18 .

\section{RESULTADOS}

A amostra foi constituída por 37.099 episódios atendidos no SUP no período em estudo, entre os quais $19.662(53 \%)$ das crianças eram do sexo masculino. Segundo a classificação adaptada do sistema de triagem de Manchester, verificou-se que 2.650 episódios eram muito urgentes (laranja), 12.820 eram urgentes (amarelos) e 21.177 eram pouco urgentes (verdes). Essas três categorias somaram 36.647 episódios (98,8\% do total de atendimentos). Os episódios considerados não urgentes (azuis) foram 19 , que somados aos verdes resultam em 21.196 e não modificam a porcentagem de episódios pouco urgentes: $57,1 \%$ do total de episódios atendidos no SUP.

A média de idade dos pacientes foi de 5,3 anos e a mediana de 4 anos (intervalo interquartil 1-9 anos). Verificou-se que as crianças que mais foram levadas ao SUP no período em questão tinham até 12 meses (15\%) e, entre todos os episódios de urgência pediátrica, $29.082(78,4 \%)$ das crianças tinham menos de 10 anos. Também foi notória a ocorrência decrescente de episódios em relação à idade da criança, ou seja, à medida que a idade aumenta, diminui o número de episódios de urgência ocorridos (Figura 2).

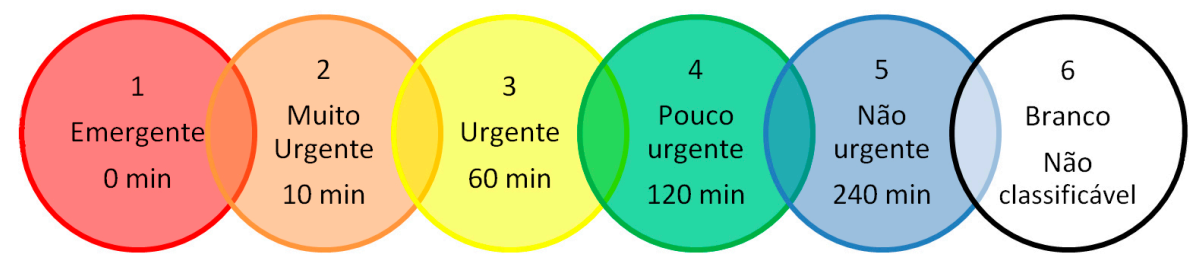

Figura 1. Classificação de prioridades para atendimento em serviço de urgência, segundo o Sistema de Triagem de Manchester, adaptado para Portugal. Os minutos significam o tempo aproximado que o paciente pode esperar pelo atendimento.

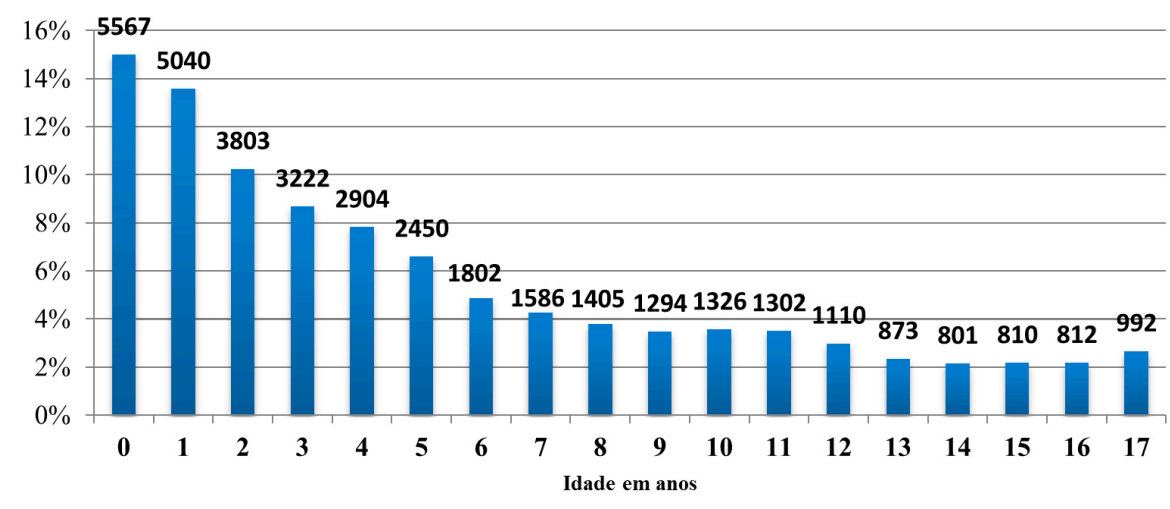

Figura 2. Número de episódios, segundo a idade, atendidos no Serviço de Urgência Pediátrica de um hospital geral e distrital da Área Metropolitana de Lisboa, Portugal, durante o ano de 2012. Porcentagens sobre o número total de episódios atendidos $(n=37.099)$. 
No que concerne às características relacionadas com a afluência ao SUP, a média diária foi de 101,6 episódios. Quanto ao dia da semana, verificou-se ser uma amostra muito homogênea, com discretas diferenças entre o dia de maior afluência, a segundafeira (16\%) e o de menor, o sábado (12\%). Os episódios verdes (pouco urgentes) ocorreram mais ao fim de semana, de madrugada e de manhã, e sobretudo no sexo feminino. Já os episódios amarelos e laranjas (urgentes e muito urgentes) ocorreram mais durante a semana, nos horários entre as $12 \mathrm{~h}-14 \mathrm{~h}$ e as $18 \mathrm{~h}-21 \mathrm{~h}$, e mais no sexo masculino (Tabela 1).

Em relação à hora de entrada no serviço de urgência podemos ver uma distribuição com algumas oscilações. A afluência começa a aumentar a partir das 10 horas da manhã, abranda ligeiramente à hora do almoço, entre as 13 e as 14 horas, e volta a subir desde então para só sofrer uma quebra a partir das 23 horas. É ainda de referir que a recorrência ao serviço de urgência diminui drasticamente durante a noite. Se analisarmos simultaneamente o dia de semana e a hora de entrada dos episódios no SUP, vemos que as horas de maior afluência se mantiveram constantes ao longo de todos os dias da semana, não havendo um dia com uma hora mais prevalente (Figura 3).

Quanto à média de idades, as crianças com episódios urgentes (amarelos) tinham, em média, uma idade superior $(9,3 \pm 0,24$ anos) à dos indivíduos com os outros tipos de episódios $(\mathrm{p}<0,001)$.

Tabela 1. Relações entre o tipo de episódio segundo o critério de urgência (considerando os episódios verdes, amarelos e laranjas*, que compõem 98,8\% do total de episódios atendidos) e as variáveis dia da semana, hora e sexo. Serviço de Urgência Pediátrica de um hospital geral e distrital da Área Metropolitana de Lisboa, Portugal, ano de 2012.

\begin{tabular}{|c|c|c|c|c|}
\hline \multirow[b]{2}{*}{ Variáveis } & \multicolumn{3}{|c|}{ Tipos de episódio* } & \multirow[b]{2}{*}{$p^{\dagger}$} \\
\hline & $\begin{array}{l}\text { Verdes } \\
\mathrm{n}(\%)\end{array}$ & $\begin{array}{c}\text { Amarelos } \\
\text { n (\%) }\end{array}$ & $\begin{array}{c}\text { Laranjas } \\
\text { n (\%) }\end{array}$ & \\
\hline \multicolumn{5}{|l|}{ Dia da semana } \\
\hline Fim de semana & $5963(28,2)$ & $3073(24)$ & $662(25)$ & \multirow{2}{*}{$<0,001$} \\
\hline Dia de semana & $15214(71,8)$ & $9747(76)$ & $1988(75)$ & \\
\hline \multicolumn{5}{|l|}{ Horário } \\
\hline $1 \mathrm{~h}-10 \mathrm{~h}$ & $5201(24,6)$ & $2394(18,7)$ & $373(14,1)$ & \multirow{2}{*}{$<0,001$} \\
\hline $12 \mathrm{~h}-14 \mathrm{~h}$ e $18 \mathrm{~h}-21 \mathrm{~h}$ & $8624(40,7)$ & $5854(45,7)$ & $1281(48,3)$ & \\
\hline \multicolumn{5}{|l|}{ Sexo da criança } \\
\hline Feminino & $10673(50,4)$ & $5846(45,6)$ & $1055(39,8)$ & \multirow{2}{*}{$<0,001$} \\
\hline Masculino & $10504(49,6)$ & $6974(54,4)$ & $1595(60,2)$ & \\
\hline
\end{tabular}

* Classificação de Manchester: verde, episódio pouco urgente; amarelo, urgente; laranja, muito urgente. Número total de atendimentos ocorridos no período: 37.099.Número total de episódios verdes, amarelos e laranjas ocorridos no período: 36.647.

† Teste do qui-quadrado com respectivo ajuste residual.

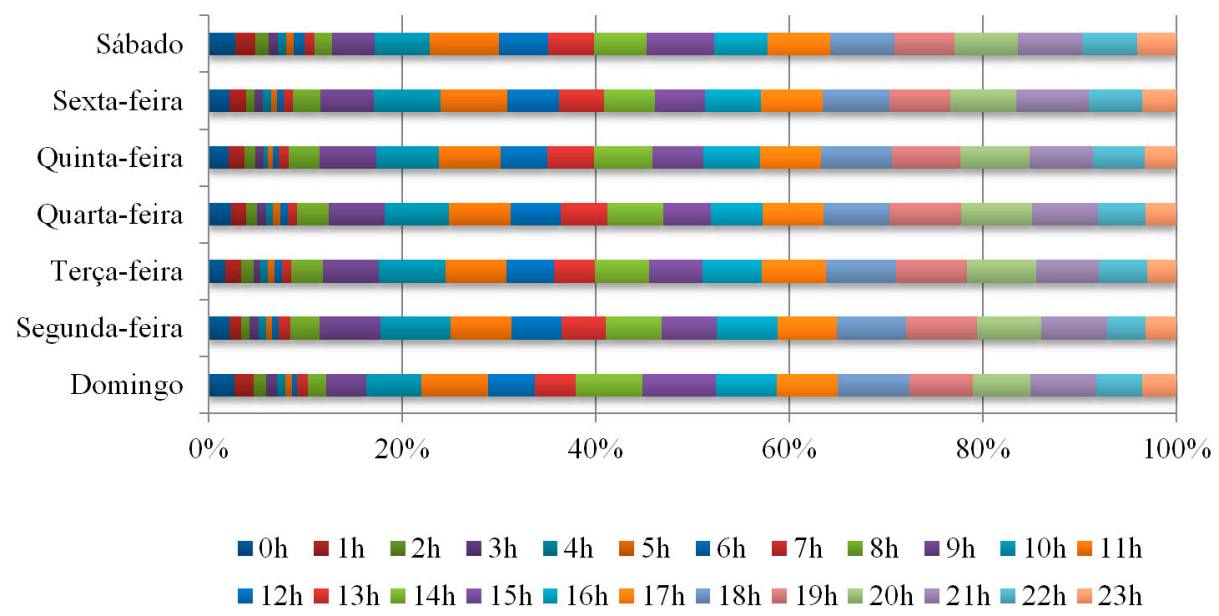

Figura 3. Distribuição segundo hora do dia e dia da semana, de 37.099 episódios atendidos no Serviço de Urgência Pediátrica de um hospital geral e distrital da Área Metropolitana de Lisboa, Portugal, durante o ano de 2012. 
Em relação aos MCDT, verificou-se a sua realização em $7.792(21 \%)$ dos episódios. Observou-se que os MCDT realizaram-se mais nos episódios amarelos e laranjas, às quartas e sextas-feiras, nos intervalos de tempo entre $12 \mathrm{~h}-14 \mathrm{~h}$ e $17 \mathrm{~h}-19 \mathrm{~h}$ e nas crianças entre os 10 e os 15 anos (Tabela 2).

Tabela 2. Relação entre variáveis e a utilização de meios complementares de diagnóstico e terapêutica em atendimentos realizados no Serviço de Urgência Pediátrica de um hospital geral e distrital da Área Metropolitana de Lisboa (considerando os episódios verdes, amarelos e laranjas*, que compõem 98,8\% do total de episódios atendidos). Lisboa, Portugal, 2012.

\begin{tabular}{|c|c|c|c|}
\hline Variáveis & $\begin{array}{l}\text { Número de } \\
\text { episódios }\end{array}$ & $\begin{array}{c}\text { Realização } \\
\text { de } \mathrm{MCDT}^{+} \\
\text {n (\%) }\end{array}$ & $\mathrm{p}^{\ddagger}$ \\
\hline \multicolumn{4}{|l|}{ Categoria do episódio* } \\
\hline Verde & 21.177 & $2.293(10,8)$ & \multirow{2}{*}{$<0,001$} \\
\hline Amarelo ou laranja & 15.470 & $5.444(35,2)$ & \\
\hline \multicolumn{4}{|l|}{ Dia da semana } \\
\hline Quarta-feira e sexta-feira & 10.372 & $2.549(24,6)$ & \multirow{2}{*}{$<0,001$} \\
\hline Outros dias & 26.727 & $5.243(19,6)$ & \\
\hline \multicolumn{4}{|l|}{ Hora } \\
\hline $12 \mathrm{~h}-14 \mathrm{~h}$ e $17 \mathrm{~h}-19 \mathrm{~h}$ & 13.264 & $3.166(23,9)$ & \multirow{2}{*}{$<0,001$} \\
\hline Outros períodos & 23.835 & $4.626(19,4)$ & \\
\hline \multicolumn{4}{|l|}{ Idade } \\
\hline Entre 11 e 15 anos & 4.896 & $1.979(40,4)$ & \multirow{2}{*}{$<0,001$} \\
\hline Outras idades & 32.203 & $5.813(18,0)$ & \\
\hline
\end{tabular}

* Classificação de Manchester: verde, episódio pouco urgente; amarelo, urgente; laranja, muito urgente.

Número total de atendimentos ocorridos no período: 37.099.

Número total de episódios verdes, amarelos e laranjas ocorridos no período: 36.647.

Número total de episódios em que foram realizados meios complementares de diagnóstico e terapêutica: 7797.

${ }^{\dagger} \mathrm{MCDT}$, meios complementares de diagnóstico e terapêutica

₹ Teste do qui-quadrado com respectivo ajuste residual.
A distribuição da amostra segundo o tipo de saída (desfecho) do episódio de urgência mostrou que 98\% tiveram alta e $2 \%$ necessitaram ficar internados. $\mathrm{Na}$ comparação dos tipos de saída entre as categorias de urgência dos episódios, verificou-se que as taxas de hospitalização diferiram entre os tipos de episódio, sendo muito maiores nos episódios laranja (muito urgentes) comparados aos amarelos (urgentes) e verdes (pouco urgentes). Verificou-se que em $90,8 \%$ dos 2.650 episódios muito urgentes, $97,1 \%$ dos 12.820 urgentes e $99,4 \%$ dos 21.177 pouco urgentes os pacientes tiveram alta sem necessitar de hospitalização (Figura 4).

\section{DISCUSSÃO}

Nas últimas duas décadas, Portugal procurou nas suas políticas de saúde promover o direito de acesso aos cuidados de saúde, sobretudo diferenciados, e nesse sentido foram criadas plataformas de informação e de aumento da própria acessibilidade [16,17]. Com o aumento da oferta e maior facilidade de acesso, aumentou também a procura, gerando superlotação dos serviços de urgência, sobretudo às custas de episódios pouco urgentes $[1,18]$.

A utilização excessiva dos serviços de urgência tem tido um papel protagonista em vários estudos no âmbito da gestão de saúde, dado tratar-se de uma problemática crescente já há vários anos [19,20]. Nesse sentido, este estudo procurou contribuir um pouco mais para o conhecimento dessa realidade, tendo-se concluído que mais de metade dos episódios de urgência pediátrica eram pouco urgentes ou não eram urgentes. Estes resultados vão ao encontro de outros estudos realizados em Portugal e em outros países [5,7,21-23] em que também mais de $50 \%$ dos episódios que recorreram

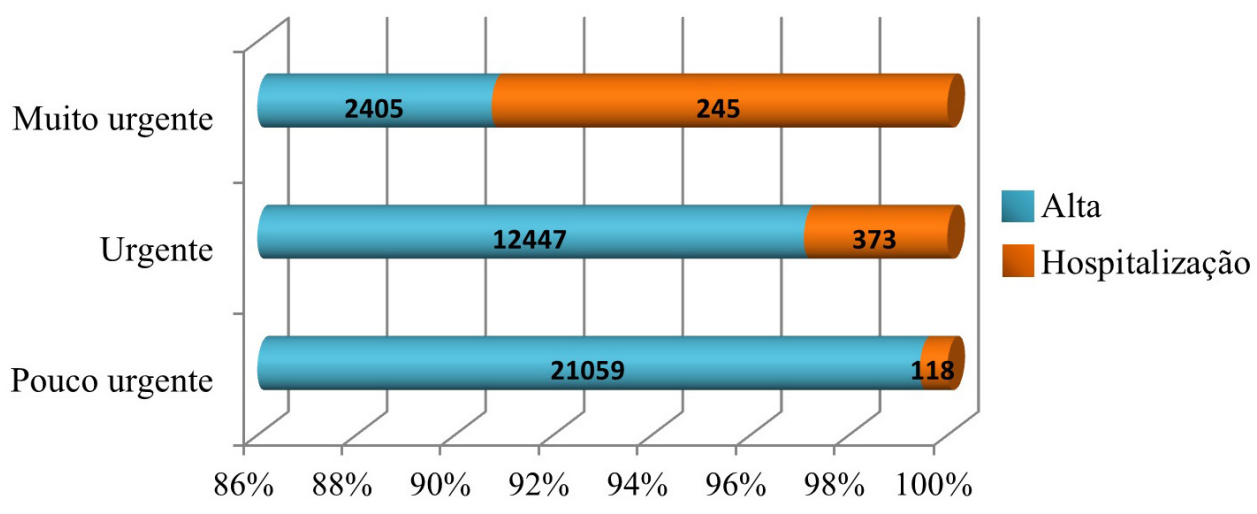

Figura 4. Relação entre o tipo de episódio e o tipo de saída, em episódios atendidos no Serviço de Urgência Pediátrica de um hospital geral e distrital da Área Metropolitana de Lisboa, Portugal, considerando somente os episódios muito urgentes, urgentes e pouco urgentes ocorridos $(n=36.647$ entre um total de 37.099) durante o ano de 2012. 
aos SUP tratavam-se de casos não urgentes [18]. A constatação da quase totalidade de altas nos episódios verdes e taxas um pouco maiores de hospitalizações nos episódios considerados urgentes e muito urgentes pela classificação de Manchester adaptada põe em evidência as vantagens desta classificação para indicar a gravidade do quadro clínico do paciente pediátrico.

Verificamos também que a maioria dos episódios coincidiu com o horário de funcionamento dos cuidados de saúde primários (dias úteis entre as $8 \mathrm{~h}$ e as $20 \mathrm{~h}$ ). Constata-se, assim, a necessidade de maior sensibilização da população a essa realidade e, consequentemente, um investimento na educação para a saúde e a aproximação das pessoas aos cuidados de saúde primários [10,17,21], que devem ser o primeiro contato da comunidade com o sistema de saúde. No que diz respeito aos episódios pouco urgentes, verificou-se uma afluência predominantemente em dias e horas em que os ambulatórios oferecem serviços mais reduzidos, quer em número quer em horário, o que permite concluir, tal como em outros estudos [6,22,24,25], que deve ser ponderada uma maior flexibilidade de horário, de forma a adequar-se a oferta às necessidades da população. Também estes resultados vão ao encontro de outros que demonstram que a procura de cuidados de saúde a nível dos SUP tem picos assistenciais, variando ao longo do dia e da semana [26-29].

No que diz respeito aos MCDT, hoje em dia utilizados como referência estatística hospitalar para medir a produção dos serviços, é também importante perceber de que modo são utilizados no SUP. Importa ainda frisar que segundo alguns estudos [23,30,31], uma grande parte da procura indiscriminada aos serviços de urgência é decorrente da fácil acessibilidade e da vasta oferta de recursos especializados, quer humanos quer técnicos, como os MCDT, mesmo quando a sintomatologia não os justifica. Ou seja, apesar deste estudo demonstrar que a maioria dos episódios não realizou qualquer MCDT, já que esta é uma decisão do médico, pode ter sido este o motivo para ida à urgência em muitos casos.

Reforça-se a necessidade de acompanhamento periódico por parte do médico assistente na rede de cuidados primários de saúde, sobretudo na idade pediátrica, assim como a necessidade de uma melhor comunicação entre os vários níveis de cuidados, para evitar que situações que poderiam ser resolvidas no ambulatório acabem nos hospitais. Essa distorção pode causar prejuízos à eficácia e à qualidade do atendimento do serviço de urgência, assim como um consumo de recursos humanos, tecnológicos e financeiros, cada vez mais difíceis de conduzir [25]. Os custos gerados pela utilização indevida dos serviços de urgência poderiam ser utilizados na promoção dos cuidados de saúde primários, incluindo a melhoria de infraestruturas em quantidade e em qualidade, assim como melhoria ou criação de programas de aproximação entre o doente e o seu médico assistente.

Muitas são as medidas sugeridas em diversos estudos [6,23-25] para resolver este problema. Algumas, como a telemedicina como meio de triagem pré-hospitalar, já existem em Portugal. No entanto, outras medidas, como a existência de pediatras nos cuidados de saúde primários, ou simplesmente a correta utilização dos recursos por parte da população, são muito difíceis de implementar. Isto porque essas medidas exigem por um lado uma estrutura de cuidados sólida, bem organizada, que funcione corretamente e com uma rede de referenciação hospitalar subjacente [7], mas sobretudo exigem da população o cumprimento da cadeia de atendimento. Por outro lado, a saúde é um bem de especificidade única, de tal ordem que, a par do direito inequívoco dos cidadãos de a ele recorrer, torna qualquer medida de promoção da racionalização da procura difícil de implementar.

Assim, é necessário um reforço nas estratégias educacionais à comunidade, que muitas vezes passa apenas por dar a conhecer os recursos existentes. Alguns autores afirmam que esforços educacionais junto aos cuidadores podem ter efeitos sobre comportamentos mais responsáveis na utilização de serviços de saúde [5]. Foi inclusive possível demonstrar que os serviços prestados por assistentes sociais, com o intuito de ajudar os doentes a encontrar alternativas adequadas de prestação de cuidados de saúde em nível primário, por meio de aconselhamento, encaminhamento e articulação com recurso à comunidade, têm apresentado resultados bastante satisfatórios na taxa de reincidência ao serviço de urgência de crianças com necessidades pouco urgentes [32].

Com base em medidas já descritas e com expectativa de resultados a longo prazo, este estudo sugere a necessidade de desenvolver campanhas de sensibilização junto à comunidade, por meio de palestras de esclarecimento, realizadas por pediatras, tanto nos centros de saúde como nas escolas e creches, sobre as doenças mais frequentes, medidas de atuação em caso de doença e sobretudo esclarecimento de dúvidas com que os pais muitas vezes se deparam e que podem motivar idas desnecessárias ao SUP. Por outro lado, essas ações intersetoriais promovem um maior envolvimento da comunidade com os cuidados 
de saúde. Quanto aos grupos mais vulneráveis ou de risco, que têm maior dificuldade de acesso à informação, poderia ser feito um esforço para ir ao encontro deles, salvaguardando-se assim os princípios de equidade e qualidade do Serviço Nacional de Saúde. Aqui a intervenção deve ser priorizada, avaliando-se as necessidades reais da população, de forma a intervir onde realmente existem maiores carências, através, por exemplo, de equipas de apoio domiciliar.

Em suma, este estudo concluiu que mais de metade das crianças que utilizaram o SUP do hospital em causa apresentavam situações pouco urgentes, grande parte dos atendimentos ocorreu durante o horário de funcionamento dos centros de saúde e praticamente todas tiveram alta para o domicílio, com indicação para seguimento pelo médico assistente. Apesar de ser necessária maior investigação nesta área, pensamos que o presente estudo terá contribuído para aumentar o conhecimento da problemática da utilização excessiva dos SUP e para mostrar que é necessário pôr em prática medidas que visem à melhoria da utilização dos recursos, contribuindo para a prestação de cuidados de saúde de qualidade.

\section{NOTAS}

\section{Apoio financeiro}

Este estudo não recebeu apoio financeiro de fontes externas.

Declaração de conflito de interesses

Os autores declaram não haver conflitos de interesses relevantes ao conteúdo deste estudo, informam ter tido acesso a todos os dados obtidos e assumem completa responsabilidade pela integridade dos resultados.

\section{REFERÊNCIAS}

1. Goodell S, DeLia D, Cantor JC. Emergency department utilization and capacity. Synth Proj Res Synth Rep. 2009; $17: 1-5$.

2. Ministério da Saúde (BR). Despacho no 9871/2010. DR Série II. 2010;112: 32123.

3. Januário L. A urgência pediátrica na encruzilhada. Rev Saúde Infant. 2010;32(2):51.

4. Ministério da Saúde (BR). Proposta de requalificação das urgências: esclarecimentos adicionais. Brasília: Ministério da Saúde; 2007. Comunicado no 1.

5. Phelps K, Taylor C, Kimmel S, Nagel R, Klein W, Puczynski S. Factors Associated With Emergency Department Utilization for Nonurgent Pediatric problems. Arch Fam Med. 2000;9(10):1086-92. https://doi.org/10.1001/archfami.9.10.1086

6. Brousseau DC, Bergholte J, Gorelick MH. The effect of prior Interactions with a primary care provider on nonurgent pediatric emergency department use. Arch Pediatr Adolesc Med. 2004;158(1):78-82. https://doi.org/10.1001/archpedi.158.1.78

7. Ko M, Lee Y, Chen C, Chou P, Chu D. Prevalence of and Predictors for Frequent Utilization of Emergency Department - A Population-Based Study. Med J. 2015;94 (29):1-9. http://dx.doi.org/10.1097/MD.0000000000001205

8. Contrato-Programa 2012. Metodologia para a definição de preços e fixaçãoo de objectivos. Administração Central do Sistema de Saúde, IP. Lisboa; 2012.

9. Observatório Português dos Sistemas de Saúde. Relatório de primavera: duas faces da saúde. [Lisboa]: OPSS; 2013.

10. Contrato-Programa 2014. Metodologia para definição de preços e fixação de objectivos. Administração Central do Sistema de Saúde, IP. Lisboa; 2013.

11. Portal da codificação GDH [Internet]. Lisboa: Administração Central do Sistema de Saúde; 1989 [uptodate 2002 Apr 2; cited 2016 Oct 14]. Rogério Santos. Hospitais distritais. Available from: http://portalcodgdh.min-saude.pt/index.php/ Página_principal

12. Regulamento interno do Centro Hospitalar Barreiro Montijo.[Lisboa]; 2013.

13. Pinheiro AP. Referenciação à Urgência Pediátrica do Hospital S. Teotónio - Viseu. Rev Port Clín Ger. 2008;24(67):1-8.

14. Santos AP, Freitas P, Martins HMG. Manchester triage system version II and resource utilization in emergency department. Emerg Med J. 2010;10:1-5.

15. Ministério da Saúde (BR). Despacho no 5414/2008. DR Série II. 2008;42:8083-5.

16. Mccoy HV, Kipp CW, Ahern M. Reducing older patients' reliance on the emergency department. Soc Work Health Care. 1992;17(1):23-7. https://doi.org/10.1300/J010v17n01_02

17. Saúde procuram-se novos caminhos. In: Observatório Português dos Sistemas de Saúde. Relatório de primavera. [Lisboa]: OPSS; 2016. p. 21-56.

18. Northington WE, Brice JH, Zou B. Use of an emergency department by nonurgent patients. Am J Emerg Med. 2005;23(2): 131-7. https://doi.org/10.1016/j.ajem.2004.05.006 
19. Weiss AJ, Wier LM, Stocks C, Blanchard J. Overview of Emergency Department Visits in the United States, 2011. Healthcare cost and utilization project. Healthcare Cost and Utilization Project (HCUP) Statistical Briefs Rockville (MD): Agency for Healthcare Research and Quality (US); 2006.

20. Caldeira T, Santos G, Pontes E, Dourado R, Rodrigues L. O dia-a-dia de uma Urgência Pediátrica. Acta Pediatr Port. 2006;1(37):1-4.

21. Grassino EC, Guidi C, Monzani A, Di Pietro P, Bona G. Access to pediatric emergency departments in Italy: a comparisson between immigrant and italian patients. Ital J Pediatr. 2008;35(3):1-5.

22. Melo EO. Acessibilidade/utilização do serviço de urgência do hospital pediátrico de Coimbra. Rev Enferm (Lisboa). 1999;3:59-62.

23. Ministério da Saúde (BR). Portaria no 147/2016. DR Série I. 2016;97:1616-9.

24. Pileggi C, Raffaele G, Angelillo IF. Pediatric utilization of an emergency department in Italy. Eur J Public Health. 2006;16(3):563-9.

25. Dieguez JKI, Candela RC, Velázquez SH, Prieto AG, Fernández LG, De Llano JMA. Estudio de la demanda de asistencia pediátrica en el servicio de urgencias de un hospital general de segundo nivel. Bol Pediatr. 2004;44(187):20-5.

26. Fisher L, Begley C, Giardino A. The impact of extend hours Primary Care on Emergency Department Use Among Medicaid/SCHIP Enrollees in Houston. School of Public Health, University of Texas. Houston; 2006.

27. Direção-Geral da Saúde (PO). Divisão de Saúde Materna, Infantil e dos Adolescentes. Saúde Materno-Infantil. Rede de Referenciação Materno-Infantil. Lisboa: Direção-Geral da Saúde; 2001.

28. Santos MI, Rosário F, Figueiredo C. Padrões temporais de recurso à Urgência Pediátrica: pistas importantes para um modelo de predição da afluência. Acta Ped Port. 2010;41(4):166-70.

29. Scoble M. Implementing triage in children's assessment unit. Nurs Stand. 2004;18(34):41-4.

30. Antón MD, Pe-a JC, Santos R, Sempere E, Martínez J, Pérula LA. Demanda inadecuada en un servicio de urgencias pediátrico hospitalario: factores implicados. Med Clin (Barc). 1992;99:743-6.

31. Fernández P, Lasoasa JC, Hernando FJM, Santervas JF. Estudio de la demanda urgente de asistencia pediátrica en el servicio de urgencias de un hospital infantil. An Esp Pediatr. 1995;42(1):27-30.

32. Farion KJ, Wright M, Zemek R, Neto G, Karwowska A, Tse S, Reid S, Jabbour M, Poirier S, Moreau KA, Barrowman N. Understanding Low-Acuity Visits to the Pediatric Emergency Department. PLoS One. 2015 June 17;10(6):e0128927. https://doi.org/10.1371/journal.pone.0128927 